MODELING, IDENTIFICATION AND CONTROL, 1983, VOL. 4, NO. 1, 25-31

doi:10.4173/mic.1983.1.2

\title{
Resource allocation by convex monotonous programming-application to an OTEC energy production platform
}

\author{
K. M. MJELDE† and S. FJELD +
}

Keywords: Resource allocation, non-linear programming, convex programming, structural optimization.

\begin{abstract}
A problem is considered of the minimization of a convex and monotonically increasing cost function subject to an upper bound of a convex and monotonically decreasing failure probability function. An algorithm is defined for the parametrical solution of the problem with respect to the selected failure probability level; the algorithm is applicable to problems with cost and failure probability functions that are not necessarily separable, thus extending a previously given algorithm for separable functions. A condition is given for the relaxation of the convexity assumption on the functions, and it is observed that the problem of the minimization of the failure probability subject to an upper bound on the total cost is solved simultaneously with the given problem. An analytically solvable numerical example is given and an application to the optimization of an OTEC energy production platform is briefly described, with reference to a complete description; it is concluded that the given extension of the algorithm to non-convex functions is important in this application.
\end{abstract}

\section{Introduction}

The work described in this paper was applied in the optimization of an OTECplatform, used for offshore thermal energy conversion by harnessing the thermal gradients between the ocean surface and great depths. The work is described in detail in Fjeld, Stokke, Rønning, Mjelde, and Tvedt (1981). Several related aspects, including a model for inspection planning, are given by Sletten, Mjelde, Fjeld and Lotsberg (1982).

The motivation of the work is the high costs incurred in satisfying the requirements of a reliable structure. This makes it desirable to study the design of the structure in terms of a mathematical model of the minimization of the cost to obtain a selected reliability; this selected reliability level being varied parametrically in order to discuss the associated change of the total cost and the change of the design.

Methods of the allocation of resources to activities, see Mjelde (1983), are applied; in particular an algorithm of Luss and Gupta (1975) is extended to the problem discussed in this paper. Following a description of the model and the associated solution algorithm, the paper gives an analytically solvable example and a brief summary of an application to the optimization of an OTEC-platform.

\section{Method of structural optimization}

A concise mathematical discussion of the formulation and the solution of the optimization problem is given in this section.

Subject to a given geometry the structure is defined by a set of design variables, denoted by $W_{1}, \ldots, W_{n}$; and vectorized by $W=\left(W_{1}, \ldots, W_{n}\right)$. A cost function $C(W)$

Received 14 September 1982.

+ Det norske Veritas, Høvik, Norway. 
is minimized subject to an upper bound on the structural failure probability $P_{f}(W)$. The resulting problem, denoted by $Q$, takes the form

$$
Q \text { : Minimize } z=C(W)
$$

subject to the constraints

$$
P_{f}(W)<P_{f}^{*}
$$

and

$$
W>0
$$

where $C(W)$ and $P_{f}(W)$ are convex and differentiable functions of $W ; C(W)$ is increasing and $P_{f}(W)$ is strictly decreasing in each component variable $W_{i}$ for $i=1, \ldots, n$, and $C(0)=0$ and $P_{f}(0) \in\langle 0,1\rangle$. The optimal objective value of $Q$ is denoted by $\bar{z}$.

Defining the marginal cost to failure probability ratio $\eta_{i}(W)$ given by

$$
\eta_{i}(W)=-\frac{\partial C}{\partial W_{i}} / \frac{\partial P_{f}}{\partial W_{i}} ; \quad i=1, \ldots, n
$$

a feasible solution $W$ of $Q$ is optimal if and only if the following Kuhn-Tucker conditions are satisfactory for a subset $A$ of $\{1, \ldots, n\}$ consisting of the indices $i$ of design variables $W_{i}$ with $W_{i}>0$

$$
\begin{aligned}
& \eta_{i}(W)=\lambda \quad \text { if } i \in A \\
& \eta_{i}(W)>\lambda \quad \text { if } i \notin A \\
& P_{f}(W)=P_{f}^{*}
\end{aligned}
$$

where $\lambda>0$ is a non-negative Langrange multiplier. The conditions state that the marginal return ratios $\eta_{i}(W)$ of all positive design variables are identical; a design variable $W_{i}$ is zero if $\eta_{i}(W)$ is not sufficiently small. If $P_{f}^{*}$ is replaced by $P$, the Lagrange multiplier $\lambda$ of the Kuhn-Tucker conditions, when considered as a function of $P$, is denoted by $\lambda(P)$. A theorem of Everett (1963) demands that $\lambda(P)$ is monotonic in the following sense

$$
P_{1}<P_{2} \Rightarrow \lambda\left(P_{1}\right)>\lambda\left(P_{2}\right)
$$

The method of solution proposed in this paper is based on the application of the latter relationship to determine a value of $\lambda$ that satisfies the Kuhn-Tucker conditions with $P=P_{f}^{*}$ : this is achieved by increasing $\lambda$ from the initial value

$$
\lambda^{0}=\underset{i=1, \ldots, n}{\operatorname{Min}} \eta_{i}(0)
$$

to a value such that $P=P_{f}(W)=P_{f}^{*}$; a variable $W_{i_{1}}$ such that $\eta_{i_{1}}(0)=\lambda^{0}$ starts to increase first; the variable $W_{i_{2}}$ starts to increase at a value $\lambda^{1}$ of $\lambda$, where

$$
\begin{gathered}
\eta_{i_{1}}(W)=\eta_{i_{2}}(W)=\lambda^{1} \\
\eta_{i}(W) \geqslant \lambda^{1} \quad \text { for } i \notin\left\{i_{1}, i_{2}\right\}
\end{gathered}
$$

where $W$ satisfies $W_{i}=0$ for $i \neq i_{1}$. As $\lambda$ is increased, variables become positive when a relationship analogous to the one given above becomes satisfied. A variable at a positive value may also be reduced to zero again, when $\lambda$ increases. The values of $\lambda$ where a variable becomes positive or a positive variable is reduced to zero, are determined numerically by the application of Newton or gradient methods. The proposed 
algorithm extends an algorithm of Luss and Gupta (1975) for the solution of a problem of the allocation of resources to activities in a case where $C(W)$ and $P_{f}(W)$ are both separable functions; the present extension being applicable to functions $C(W)$ and $P_{f}(W)$ that are not necessarily separable.

A value of $\lambda$ where at least one $W_{i}$ becomes positive or is reduced to zero is called respectively an in-point or an out-point. If the set $A=\left\{i \mid W_{i}>0\right\}$ is denoted by $A(\lambda)$ for a given value of $\lambda$, it follows that $\lambda$ is an in-out-point if and only if

$$
A(\lambda-\epsilon) \neq A(\lambda+\epsilon)
$$

for all sufficiently small values of $\epsilon>0$, assuming that there is a finite number of in-outpoints in a relevant interval of values of $\lambda$. An in-out-point satisfies the condition (1) with $W_{i}=0$ for all $i \notin A$; an out-point satisfies the additional requirement that $W_{i}=0$ for some $i \in A$; an in-point satisfies the requirement (2) as an equality for some $i \notin A$. For a given $A$ this gives a set of equations for the possible in-out-points; the point with the smallest value of $\lambda$ is selected. It follows that the in-out-points are generated in a sequence of increasing values of $\lambda$. When an in-out-point $\lambda$ with an associated $W$ such that $P_{f}(W) \leqslant P_{f}^{*}$ has been obtained, a solution of the problem $Q$ for a given value of $P_{f}^{*}$ is determined by the simultaneous solution of eqns. (1) and (3) with $W_{i}=0$ for $i \notin A$. All the previously mentioned sets of equations are solved by any convenient method, such as a Newton or a gradient method; a numerical example with analytically solvable equations is given in the following section of this paper.

It is noted that the fundamental assumption of the previously given algorithm is the condition (4); the algorithm is applicable if this condition is satisfied and if there corresponds a uniquely determined value of $W$ to each value of $\lambda$. The implication is that the applicability of the algorithm includes cases where $C(W)$ or $P_{f}(W)$ is not necessarily convex, such as the OTEC optimization given in the final section of this paper.

The algorithm solves two related problems simultaneously; the given problem $Q$ and the problem of the minimization of $P_{f}(W)$ subject to an upper bound of $C(W)$; if $\bar{z}=C(W)$ is the minimal cost for a given $P_{f}(W)=P_{f}^{*}$ it follows, from the KuhnTucker optimality conditions of the two related problems, that $P_{f}^{*}=P_{f}(W)$ is minimal for $C(W) \leqslant \bar{z}$.

It is important that the algorithm gives a sensitivity analysis of the solution of $Q$ for selected values of $P$; the optimal value of $Q$ as a function of $P$ is denoted by $h(P)$, thus

$$
\bar{z}=C(W)=h(P)
$$

The latter relationship, when combined with the economical consequences of a failure, can be used to determine an economically optimal failure probability $\boldsymbol{P}$ by the minimization of the total costs given by

$$
C_{T}=h(P)+P \cdot C_{f}
$$

where $C_{f}$ is the cost of failure. It is understood that the design variables $W_{i}$ for $i=1, \ldots, n$ of the problem $Q$ determine initial costs, and expected costs of inspection and maintenance as parts of $h(P)$. Furthermore, the cost $h(P)$ and the probability $P$ refer to a given length of time, for instance a year and a given interest rate and a given taxation system. 
The value of $P_{f}^{*}$ in the problem $Q$ must be chosen such that:

$$
P_{\boldsymbol{f}}^{*}<\bar{P}
$$

when factors such as a possible loss of human lives or public recognition are considered.

The effect of the approach is to avoid cheap elements with a high failure probability, and to avoid costly elements with a low failure probability, since such elements contradict the optimality conditions and represent respectively weak links and overdesigned elements.

\section{Numerical example}

A simple numerical example with $n=2$ is given as follows

$$
\begin{aligned}
& C(W)=\sum_{i=1}^{n} W_{i} \\
& P_{f}(W)=P_{f 0}\left[1-\sum_{k=1}^{K} r_{k}\left(\sum_{i=1}^{n} \gamma_{i k} W_{i}\right)\right]
\end{aligned}
$$

where $P_{f 0}$ is an unknown constant, $K=2$ and the other data are given by

$$
\begin{aligned}
r_{k}\left(\epsilon_{k}\right) & =s_{k} \epsilon_{k}-m_{k} \epsilon_{k}^{2} ; \quad \epsilon_{k}<s_{k} / 2 m_{k} ; \quad k=1,2 \\
\left(s_{1}, s_{2}\right) & =(1 \cdot 3,1 \cdot 0) \\
\left(m_{1}, m_{2}\right) & =(3 \cdot 0,1 \cdot 0) \\
\left(\gamma_{i k}\right) & =\left(\begin{array}{cc}
1 \cdot 0 & 0.5 \\
0.5 & 1.0
\end{array}\right)
\end{aligned}
$$

it follows that the expressions for $r_{k}\left(\epsilon_{k}\right)$ for $k=1,2$ are valid for $\epsilon_{1}<0 \cdot 217$ and $\epsilon_{2}<0 \cdot 50$.

The solution of the problem $Q$ is given in Table 1 for three values of the cost $C$.

\begin{tabular}{clll}
\hline \multicolumn{1}{c}{$C$} & $W_{1}$ & $W_{2}$ & $P_{f} / P_{f 0}$ \\
\hline 0.06 & 0.06 & 0 & 0.9037 \\
0.30 & 0 & 0.30 & 0.6625 \\
0.40 & 0 & 0.40 & 0.6200 \\
\hline
\end{tabular}

Table 1. Numerical example.

When $C$ increases from $C=0$ to $C=0.06$, the value of $W_{1}$ increases from 0 to 0.06 with $W_{2}=0$; a further increase of $C$ causes $W_{1}$ to decrease and $W_{2}$ to increase until $W_{1}$ becomes zero when $W_{2}=0.30$; if $C$ is increases in the interval $[0.30,0.40]$ the value of $W_{1}$ stays at zero and $W_{2}$ increases to $W_{2}=0 \cdot 40$. The table contains an in-point for $C=0.06$ and an out-point for $C=0.30$. 


\section{Application to an OTEC platform}

Fjeld, Stokke, Rønning, Mjelde, and Tvedt, (1981) applied the previously given algorithm to the cost-benefit analysis of the design of an OTEC platform, in terms of the following design variables

$W_{1}$ : static strength

$W_{2}$ : redundance

$W_{3}$ : fatigue strength

$W_{4}$ : in-service inspection, damage

$W_{5}$ : in-service inspection, warning

$W_{6}$ : fabrication control, gross errors

$W_{7}$ : fabrication control, fatigue

where the following normalizations are applied: $W_{i} \in[0,1]$ for $i=1,3,5,6,7$; $W_{2} \in[1,3]$ and $W_{4} \in[0 \cdot 01,1]$.

The cost is expressed as

$$
C=C_{0}\left(C_{1}+C_{2}+C_{3}+C_{4}+C_{5}\left(1+P_{f \text { local }} C_{8}\right)+C_{6}+C_{7}\right)
$$

where $C_{0}$ is the cost of some base case and $C_{t}$ are coefficients describing the cost variations by increasing efforts to improve the safety, and the probability $P_{f \text { local }}$ is defined below.

The expressions for the $C_{t}$ are

$$
\begin{aligned}
& C_{1}=A_{i} W_{i} \text { for } i=1,3,4,5,6,7 \\
& C_{2}=1+w_{2}{ }^{2 \cdot 5} / A_{2} \\
& C_{8}=A_{8}
\end{aligned}
$$

for constants $A_{1}, i=1, \ldots, 8$.

The probability of failure is expressed as

$$
P_{f}=P_{f \text { local }} \cdot \phi_{2} \cdot \phi_{4}
$$

where the probability of local failure $P_{f}$ locat is given by

$$
P_{f \text { local }}=P_{o f \text { gross error }} \cdot \phi_{6}+P_{o f \text { accident }}+P_{o f \text { static }} \cdot \phi_{1}+P_{o f \text { fatigue }} \cdot \phi_{3} \cdot \phi_{5} \cdot \phi_{7}
$$

The $P_{o f}$ are failure probabilities for four independent failure reasons in an 'initial' situation i.e. before any of the safety measures/design variables are applied.

Expressions for the $\phi_{i}$ are given in Table 2, where the $B_{i}$ for $i=1, \ldots, 7$ are constants.

The constants $A_{i}, B_{i}$ and the $P_{o f}$ in a numerical case are given in Table 3.

The Figure gives a sensitivity analysis of cost versus failure probability.

Since the failure probabilities are estimated only in a relative sense, a sensitivity analysis, such as that given in the Figure, provides important information for a decision on the total cost level of the structure: in the example of the Figure a cost above the level from 2 to 3 will only marginally decrease the failure probability.

In this particular example the applicability of the algorithm was checked by the validity of condition (4) and the unique solvability of all the equations involved for a set of initial conditions, or there was no solution; the check was performed for an increasing sequence of values of $\lambda$. This check was applied, rather than deciding on the 


\begin{tabular}{ll}
\multicolumn{1}{c}{ Design variable } & \multicolumn{1}{c}{$\phi_{i}$} \\
\hline Static strength & $\phi_{1}=10^{-B_{1} w_{1}}$ \\
Redundance & $\phi_{2}=B_{2}^{-w_{2}}$ \\
Fatigue strength & $\phi_{3}=\left(1+w_{3}\right)^{-B_{3}}$ \\
In-service & $\phi_{4}=1-B_{4} \sqrt{ } w_{4}$ \\
$\quad$ inspection, damage & \\
In-service & inspection, warning \\
Fabrication control, & $\phi_{5}=1-B_{5} \frac{1-e^{-w_{5}}}{1-e^{-1}}$ \\
gross error & $\phi_{6}=\frac{B_{6}}{\sqrt{ }\left[B_{6}{ }^{2}+w_{6}\right]}$ \\
Fabrication control, & $\phi_{7}=\frac{B_{7}}{\left(B_{7}+w_{7}\right)^{2}}$ \\
\hline
\end{tabular}

Table 2. The functions $\phi_{i}$.

\begin{tabular}{cccccclll}
\hline$i$ & 1 & 2 & 3 & 4 & 5 & 6 & 7 & 8 \\
$A_{i}$ & $0 \cdot 8$ & 16 & $0 \cdot 2$ & 10 & 10 & $0 \cdot 03$ & $0 \cdot 1$ & 10 \\
$B_{i}$ & 3 & 10 & 12 & $0 \cdot 9$ & $0 \cdot 9$ & $0 \cdot 1$ & $0 \cdot 01$ & - \\
\hline & & & & & \\
\hline $\begin{array}{c}P_{\text {of gross error }} \\
10^{-3}\end{array}$ & $\begin{array}{c}P_{\text {of accident }} \\
10^{-3}\end{array}$ & & $\begin{array}{c}P_{\text {of static }} \\
10^{-3}\end{array}$ & $\begin{array}{c}P_{\text {of fatigue }} \\
10^{-2}\end{array}$ \\
\hline
\end{tabular}

Table 3. Values of $A_{i}, B_{i}$ and $P_{o f}$.

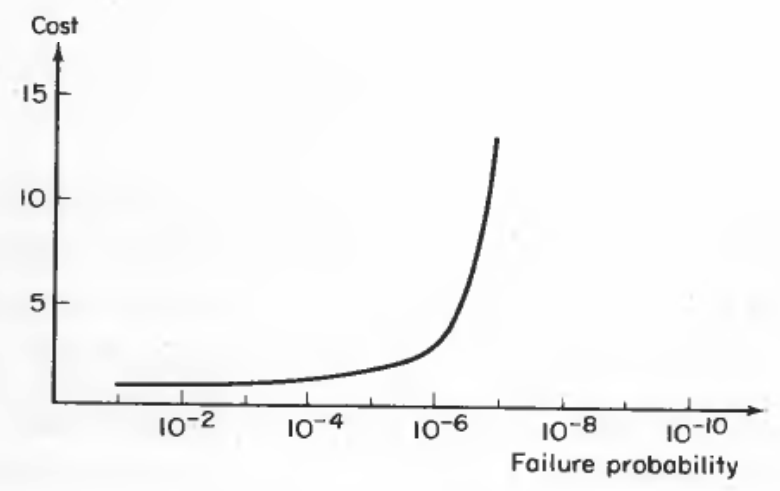

Sensitivity analysis

question of the convexity of $P_{f}(W)$ in a given region for the functions $\phi_{i}(W)$ defined in Table 2 (since the product of two convex functions is not necessarily convex, as exemplified by the product $\left(4-W_{1}\right)\left(4-W_{2}\right)$ and the points $\left(W_{1}, W_{2}\right)=(2,2),(1,3)$ $(1 \cdot 5,2 \cdot 5))$. However, for exponential functions $\phi_{i}\left(W_{i}\right)=a_{i} \exp \left(-\alpha_{i} W_{i}\right)$ the convexity of $P_{f}(W)$ is obvious, such functions appear if the relationship $\dot{\phi}_{i}{ }_{i}\left(W_{i}\right)=-\alpha_{i} \phi_{i}\left(W_{i}\right)$ is 
applicable, where $\dot{\phi}^{1}{ }_{i}$ is the derivative of $\phi_{i}$. Also note that, even for exponential functions $\phi_{i}\left(W_{i}\right)$, the convexity of $C(W)$ is not clear due to the term $C_{5} \cdot\left(1+P_{f \text { local }} C_{8}\right)$. It is therefore a particular advantage of the method proposed in this paper that cases with non-convex functions can be treated without much difficulty, subject to a confirmative check of the validity of condition (4).

The numerical example given here is only one of a large set of numerical cases discussed in Fjeld, Stokke, Rønning, Mjelde and Tvedt (1981); these cases constitute a sensitivity analysis of the problem with respect to variations of the parameters within their respective uncertainty-ranges.

\section{ACKNOWLEDGEMENT}

This work was sponsored by Giannotti \& Associates Inc. The authors appreciated the discussion of the paper at the conferences of The American Society of Civil Engineers-1982 Annual Convention, New Orleans, and The Institute of Management Sciences and The Operations Research Society of America-1982, Joint National Meeting, San Diego. Aspects of the paper were also presented at the 1982 European Petroleum Conference, London.

\section{REFERENCES}

EVERETT, M. (1963). Generalized Lagrange multiplier method for solving problems of optimum allocation of resources. Opns. Res., 11, 399-417.

Fjeld, S., Stokke, K., Rønning, B., Mjelde, K. M. and Tvedt, L. (1981). Optimal design fabrication and inspection specifications for OTEC ocean engineering systems. VERITAS, Technical Report No. BI.1047.

Luss, H., and GUPTA, S. K. (1975). Allocation of effort resources among competing activities. Opns. Res., 23, 360-366.

MJELDE, K. M. (1983). Methods of the allocation of limited resources. London, New York: John Wiley and Sons.

Sletten, R., Mjelde, K. M., Fjeld, S., and Lotsberg, I. (1982). Cptimization of criteria for design, construction and in-service inspection of offshore structures based on resource allocation techniques. Proceedings of the 1982 European Petroleum Conference, London. Organized by: Society of Petroleum Engineers U.K. Limited. Program Chairman: J. Ogrem. 